\title{
Cloud Intelligent Logistics Service Selection Based on Combinatorial Optimization Algorithm
}

\author{
Yan $\mathrm{Hou}^{1 *}$, Zhenjie $\mathrm{Cao}^{1}$, Shuling Yang ${ }^{2}$ \\ ${ }^{1}$ School of Management, Jilin Normal University, Siping 136000, China \\ ${ }^{2}$ College of foreign languages, Jilin Normal University, Siping 136000, China
}

Corresponding Author Email: hoyt1980@qq.com

https://doi.org/10.18280/jesa.520110

Received: 12 November 2018

Accepted: 5 January 2019

\author{
Keywords: \\ cloud intelligent logistics (CIL), internet \\ of things (IoT); combinatorial \\ optimization algorithm (COA), service \\ classification, service negotiation
}

\begin{abstract}
The selection of intelligent logistics service model has become an important factor in the competition of the entire social logistics industry. Using the technologies such as the Internet of Things (IoT) and cloud computing, the service platform of cloud intelligent logistics (CIL) virtualizes and accesses to the distributed physical logistics resources and logistics capabilities, and relies on its powerful processing and control capabilities to obtain the best service portfolio of CIL. The paper proposes the service combinatorial optimization algorithm (COA). Based on this, it studies the cloud intelligent logistics service and service combination method. The results show that compared with clustering algorithm and differential evolution algorithm, COA algorithm has greater superiority and stability in selection and combination of CIL service; the CIL service has the characteristics of dynamicity and diversity, heterogeneity and distribution, abstraction and similarity; the service portfolio of CIL is divided into three stages: service classification, service negotiation and optimal combination. The application of COA in the CIL selection can greatly reduce the time consumption of combined logistics service and improve the overall service quality of combinatorial service.
\end{abstract}

\section{INTRODUCTION}

With the globalization and large-scale development of economy and trade, especially the rapid development of ecommerce, the intelligent logistics not only plays one more important role in the national economy, but also becomes the bottleneck of economic development [1]. The logistics cost of China's manufacturing enterprises has remained high for a long time, bringing heavy pressure to the operation of enterprises. Meanwhile, extensive logistics management has become a bottleneck in the economic development of China and the world [2]. The intelligent logistics service model has become an important factor in improving the efficiency of logistics operations, reducing logistics costs, and increasing the competitiveness of logistics and the whole society [3-4]. Based on a variety of resources such as processors, memories, and application software on the virtualized Internet, Cloud computing technology builds a unified resource pool, unified management, and unified scheduling, then provides various computing services to users, and supports expansion according to customer needs [5]. At present, cloud computing has been widely used in office, education and search engines. With the slow development of intelligent logistics services, the emergence of cloud computing has brought about a new IoTbased, service-oriented and demand-oriented intelligent logistics model: Cloud Intelligent Logistics [6-7].

Cloud intelligent logistics has different characteristics in terms of different logistics service models, including ondemand services, virtualized cloud services, and virtual alliances [8]. CIL services operate and calculate in the form of cloud services, matching logistics tasks through intelligent logistics services on the cloud platform, and seamlessly connecting CIL services with logistics tasks [9]. Industry and academia have proposed many algorithms for CIL, in which the industrial community pays attention to the combination of intelligent logistics to optimize the language, while the academic community focuses on the automatic combination of intelligent logistics, but most of these algorithms can only solve the static service combination problem, without considering the distribution of services and some variable environmental information [10-11]. At present, the algorithms commonly used in the selection of CIL services mainly include clustering algorithms and differential evolution difference algorithms. The combinatorial optimization algorithm has been rarely applied in logistics services. Some research has found that this algorithm has excellent time performance on the logistics platform [12].

In summary, based on COA algorithm of service, this paper studies the CIL service and service combination method, and also the operation mechanism of CIL service. Then, it proposes the solution method and algorithm of COA algorithm. Finally, it verifies feasibility and superiority of the algorithm by simulation experiments.

\section{RESEARCH ON FORMAL DESCRIPTION METHOD OF CLOUD INTELLIGENT LOGISTICS SERVICE}

\subsection{Business process and operation mechanism of cloud intelligent logistics service}

Figure 1 shows the traditional integrated logistics service model. The whole process includes the order process, the receiving process and the evaluation process. The mature application of new technologies has enhanced the 
development of the intelligent logistics industry, which further brings the logistics service industry into a new era of changing [13]. Current indicators for measuring the quality of logistics services include personnel communication quality, orders release quantity, information quality, timeliness, and error handling [14]. The most prominent feature of CIL services is the combined application of virtual technology in the Internet of Things and the computing power of cloud computing, achieving the intelligent matching, intelligent combination and intelligent management of logistics service processes [15]. The operation process of CIL requires the participation of operators, providers and users. When the user issues the usage requirements, the intelligent system will automatically match the suppliers and operators, and the operators will transfer from the provider to the use [16-17]. Figure 2 shows the discovery and combination mechanism of the CIL service. Both providers and users can register services on the cloud platform. The CIL service platform will build a logistics task library based on the user description, link the CIL service library, and calculate and obtain a series of CIL services associated with demand.

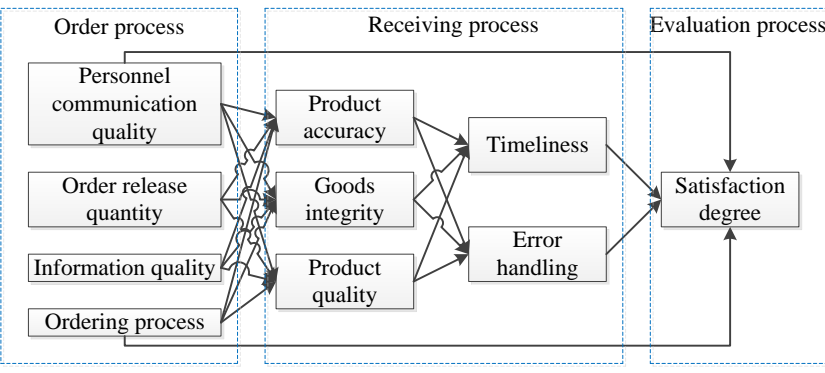

Figure 1. Traditional integrated logistics service model

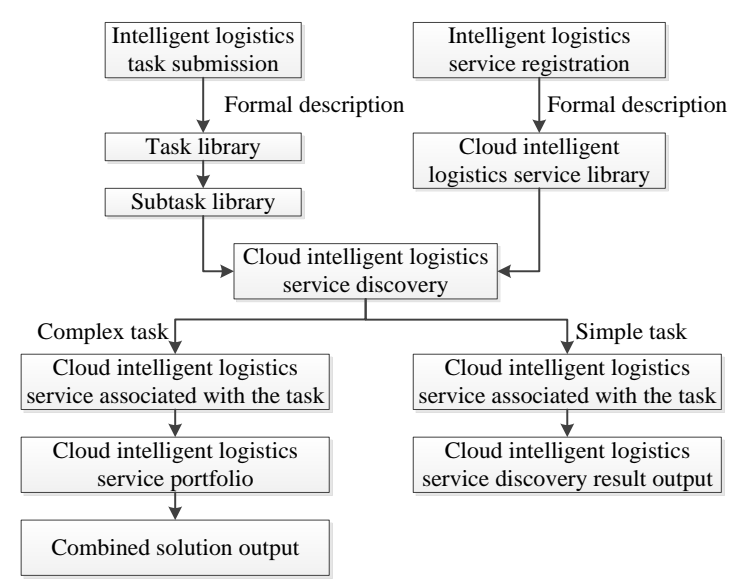

Figure 2. Discovery and combination mechanism of cloud intelligent logistics service

\subsection{Cloud intelligent logistics service modeling}

The logistics resources and logistics capabilities are processed by the cloud platform to form the logistics cloud in the form of services. The CIL service realizes two-way interaction between users and suppliers, and service selection is realized through the cloud platform. The CIL service platform builds a cloud service architecture, integrates rich information resources, realizes information sharing, meets the individualized needs of different users, and improves the operational efficiency of CIL. CIL service has the characteristics of dynamicity and diversity, heterogeneity and distribution, abstraction and similarity. The whole CIL service consists of two parts: logistics resources and logistics capabilities. Logistics resources include technical resources, human resources and user's information resources, public service resources and application system resources; and logistics capabilities include transportation capacity, distribution capacity, storage capacity, port throughput, packaging skills, and handling capabilities. Figure 3 shows the cloud ontology of intelligent logistics service. Cloud computing consists of application layer, grid computing and virtualization. The three-tier structure of cloud ontology includes infrastructure layer (Iaas), application layer (Saas) and platform layer (Paas).

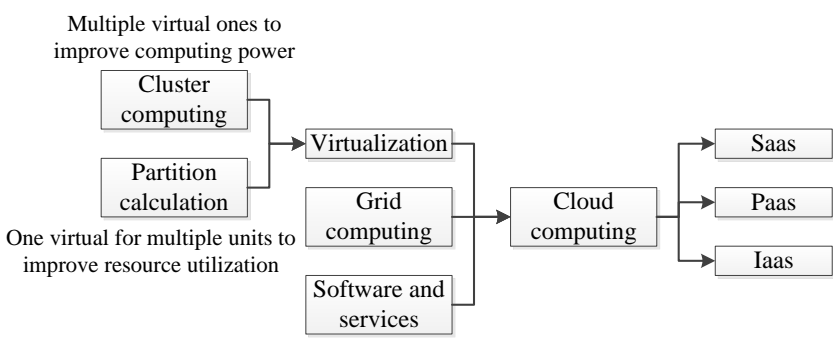

Figure 3. Intelligent logistics service cloud ontology

\section{RESEARCH ON DISCOVERY METHODS OF CLOUD INTELLIGENT LOGISTICS SERVICE}

\subsection{Discovery algorithm description of cloud intelligent logistics service}

With the promotion of CIL services, the two-way relationship between users and providers is becoming more and more apparent, but one standard, open protocol and service discovery mechanism has not been formed yet. Before the COA algorithm, clustering algorithm was most widely used in logistics service, that is, the user needs are matched with the CIL service in the clustering cluster. The increase in the number of users and data will lead to a large number of logistics tasks at the same time. When clustering algorithm is used to provide optimal CIL services for the huge logistics tasks, the matching time complexity and matching efficiency are far from enough. The quality of service matching is directly related to the quality of CIL service. The use of COA algorithm can find the extreme value of service matching and provide the optimal solution. The relevant information and the ServiceProfile of all services are extracted from the CIL platform parser, the category and attribute information in the ServiceProfile are identified and matched with the service type, and the system provides the optimal service matching degree according to the COA algorithm.

\subsection{Experiment and results analysis}

The COA algorithm and clustering algorithm were applied in the comparative experiments, to verify the superiority of COA algorithm. In this experimental environment, four ASUS machines were used to build the Haddoop cloud platform, in which one was set as the namenode service node, and the other three were set as the datanode service nodes. Table 1 lists the data set of different file sizes, record counts, and data blocks set separately. set. In the same experimental environment, the precision ratio of the $\mathrm{COA}$ and the clustering algorithm under 
different node conditions were compared. Both algorithms used a single operation, and the starting point is the designated cloud logistics service.

Figure 4 shows the comparison of the running time of single node between these two algorithms, indicating clearly that the time spent on the two algorithms varies slightly with the small data set; when the data set file is over $1.15 \mathrm{G}$, the COA algorithm takes significantly less time than the clustering algorithm. Figure 5 shows the comparison for the running time between different nodes. It can be seen that the time spent on a single task is inversely proportional to the number of nodes; increasing the number of nodes can improve the processing efficiency of the COA algorithm. As the data set increases, the same number of nodes takes longer time. Figure 6 shows the experiment results of the acceleration ratio. The acceleration ratio means the ratio of single node operation and multi-node operation. It can be seen from the figure that the acceleration ratio of data set $\mathrm{E}$ is proportional to the number of nodes, and those of the other four data sets are kept constant.

Figure 7 shows the comparative experiment results of the precision ratio. When the number of nodes is 10 , the precision of the clustering algorithm is $95.7 \%$, and that of the COA algorithm is $96.4 \%$. With the number of nodes increasing, the precision of both algorithms increases, but that of the COA algorithm is higher. When the number of nodes increases to 40 , the precision of both algorithms is 1, indicating that the precision increases with the number of nodes. Figure 8 shows the comparative results of the response time. It can be seen that the response time of both algorithms increases with the increase of the number of cloud services, but that of the COA algorithm is shorter, which reflects the advanced nature and high energy efficiency of the COA algorithm.

Table 1. Experimental data

\begin{tabular}{cccc}
\hline $\begin{array}{c}\text { Data } \\
\text { set }\end{array}$ & $\begin{array}{c}\text { Data file } \\
\text { size }\end{array}$ & $\begin{array}{c}\text { Record } \\
\text { number }\end{array}$ & $\begin{array}{c}\text { Data blocks } \\
\text { number }\end{array}$ \\
\hline A & $45 \mathrm{M}$ & 2585416 & 1 \\
B & $532 \mathrm{M}$ & 30086336 & 9 \\
C & $1.15 \mathrm{G}$ & 72568532 & 20 \\
D & $2.10 \mathrm{G}$ & 153771327 & 35 \\
E & $2.85 \mathrm{G}$ & 194781944 & 42 \\
\hline
\end{tabular}

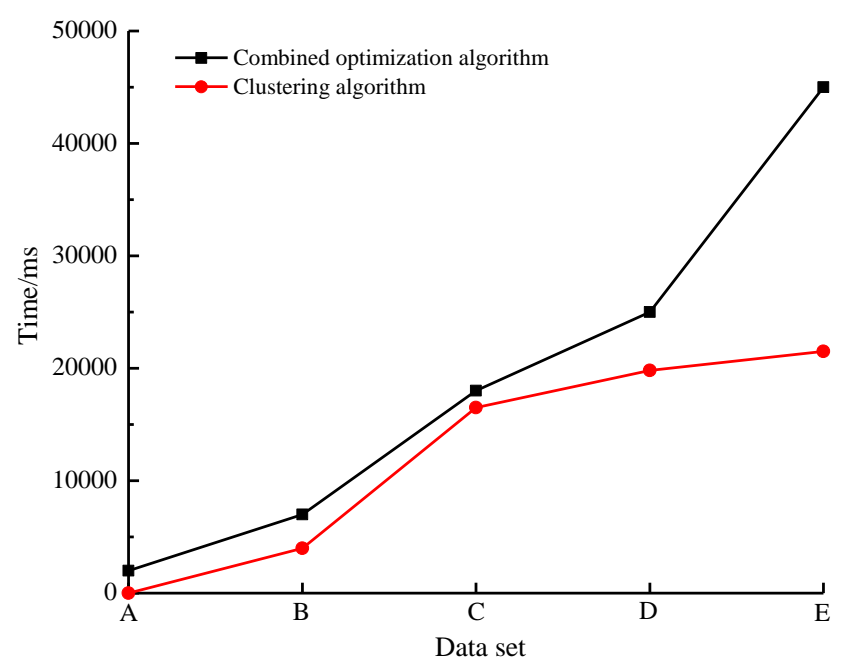

Figure 4. Comparison chart of two-node running time

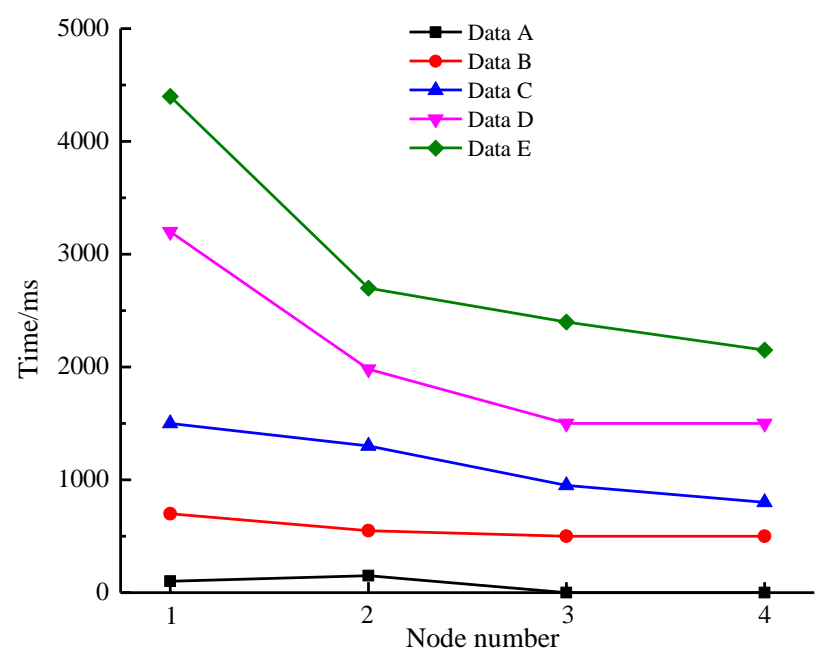

Figure 5. Comparison of running time of different nodes

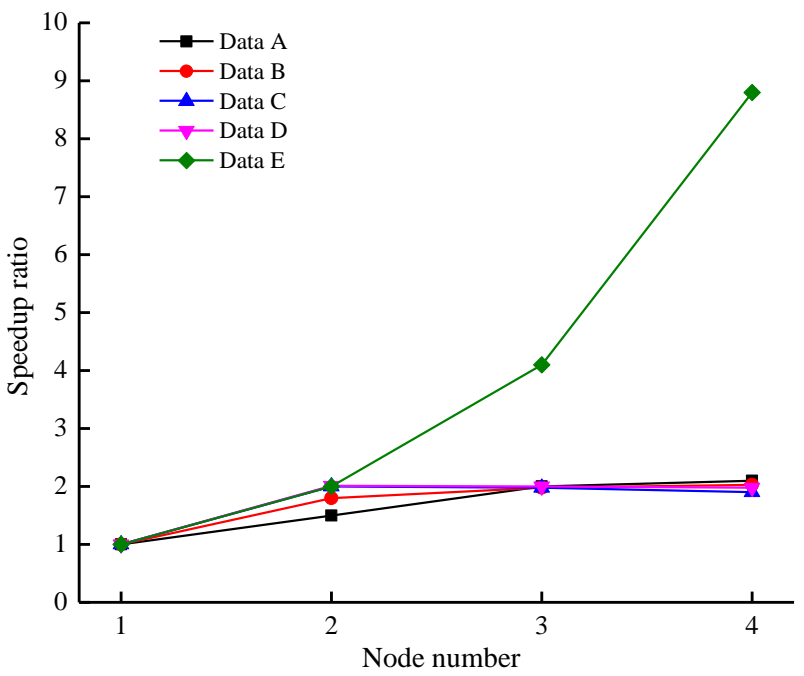

Figure 6. Acceleration ratio experiment result chart

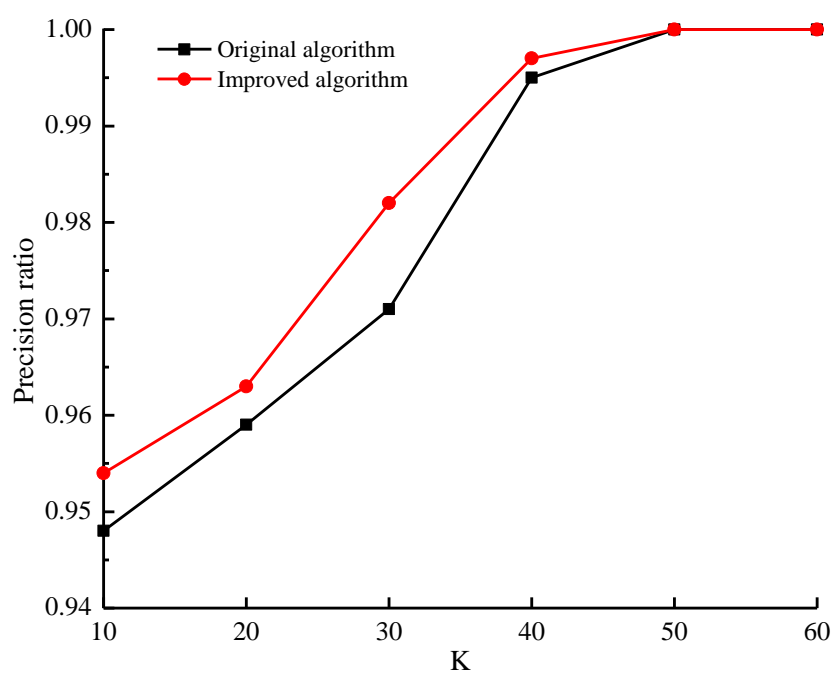

Figure 7. Calibration rate comparison test result chart 


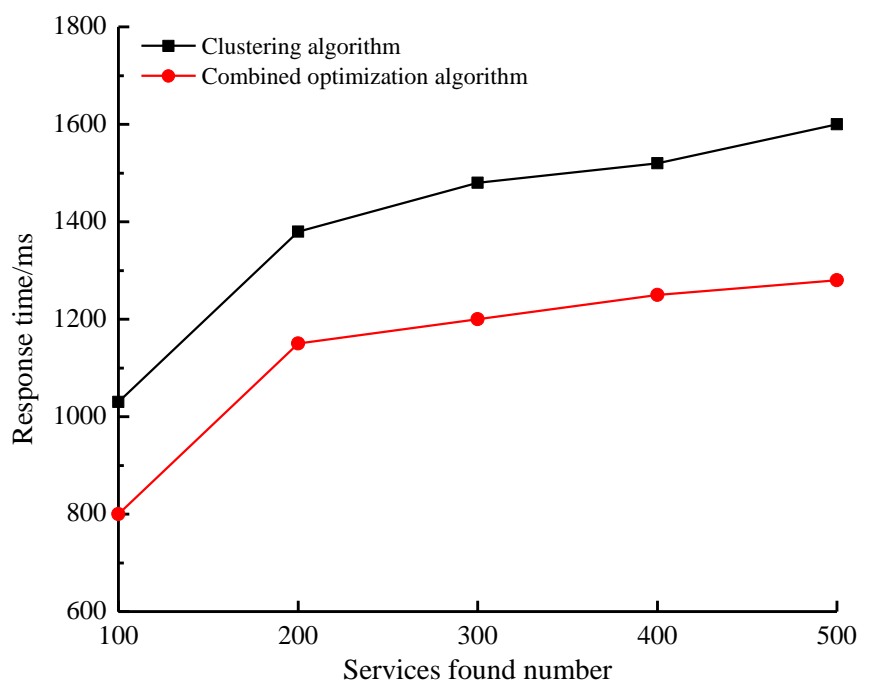

Figure 8. Response time comparison experiment result chart

\section{RESEARCH ON THE COMBINATION METHOD OF CLOUD INTELLIGENT LOGISTICS SERVICE}

\subsection{Cloud intelligent logistics service combination process and method}

When a user or provider submits a complex logistics service request, it is not enough to rely solely on the simple CIL service. For this, the complex logistics service needs to be broken down into multiple simple sub-services, and each subservice will have different requirements for cost, time and resource, which require the integration of sub-service resources to achieve optimal logistics resources. The CIL service portfolio has the characteristics of logistics path selection variety, cloud environment dynamics, variability and large amount of information. The CIL service portfolio is divided into three stages: service classification, service negotiation and optimization combination. For the optimal combination, the delivery time, running cost, reliability and credibility objective function of CIL service should be constructed. The COA algorithm is a simple and effective global optimization algorithm, which is effective, convergent and robust in solving the CIL service. Its specific operation steps include the population initialization, mutation operation, cross operation, selection operation and termination operation. Previously, the commonly used algorithm was the cloud differential evolution (DE) algorithm, which requires several iterations before obtaining the optimal solution at the low efficiency.

\subsection{Experimental simulation and results analysis}

By comparing the COA algorithm and the cloud DE algorithm, this section solves the multi-objective optimization problem of CIL service portfolio. In this experiment, eight Asus machines were used as the cloud platform, one for the namenode service node, the other seven for the datanode service node, and a total of 13 optimization functions were selected for the simulation experiment. Figure 9 shows the comparison for the average running time of the test functions between the two algorithms. It can be seen that the average running time of the COA algorithm is shorter when the multimodal function is used; In general, the COA algorithm has more obvious advantages. Figure 10 shows the comparison for the running time of the test functions between different nodes. It can be seen that the running time of the optimization functions $3,4,12$, and 13 decreases as the number of nodes increases; when the number of nodes reaches 6 , the running time does not change. Figure 11 shows the comparative results of the acceleration ratio. The acceleration ratio is the ratio of the single node to the multi-node running time, which is used to measure the service combination effect. It can be clearly seen that when the number of nodes is less than 5 , the acceleration ratio increases with the number of nodes; when the number of nodes is greater than 5 , the acceleration ratio is reduced, but not significantly, which reflects the stability and good scalability of the COA algorithm.

In order to verify the performance of the COA algorithm in simulation experiment, the Pareto frontier TSC $\left(\mathrm{A}_{1}, \mathrm{~A}_{2}\right)=0$ or 1 were defined, and then the Pareto frontier obtained by the optimal solution using the simulation experiment is expressed as:

$\operatorname{TSC}\left(A_{1}, A_{2}\right)=\frac{\left|\left\{\mathrm{a}_{2} \in \mathrm{A}_{2} \mid \exists \mathrm{a}_{1} \in \mathrm{A}_{1}, \mathrm{a}_{1} \geq \mathrm{a}_{2}\right\}\right|}{\left|\mathrm{A}_{2}\right|}$

where, $\mathrm{A}_{1}$ is the COA algorithm, and $\mathrm{A}_{2}$ is the cloud DE algorithm; $a_{1}$ and $a_{2}$ are the number of sub-tasks for these two algorithms respectively. Figure 12 shows the simulation results of the improved service combination optimization algorithm. By analyzing the mean and standard deviation of the simulation results, it is found that the COA algorithm is closer to the true Pareto frontier, that is, it's better than the cloud DE algorithm.

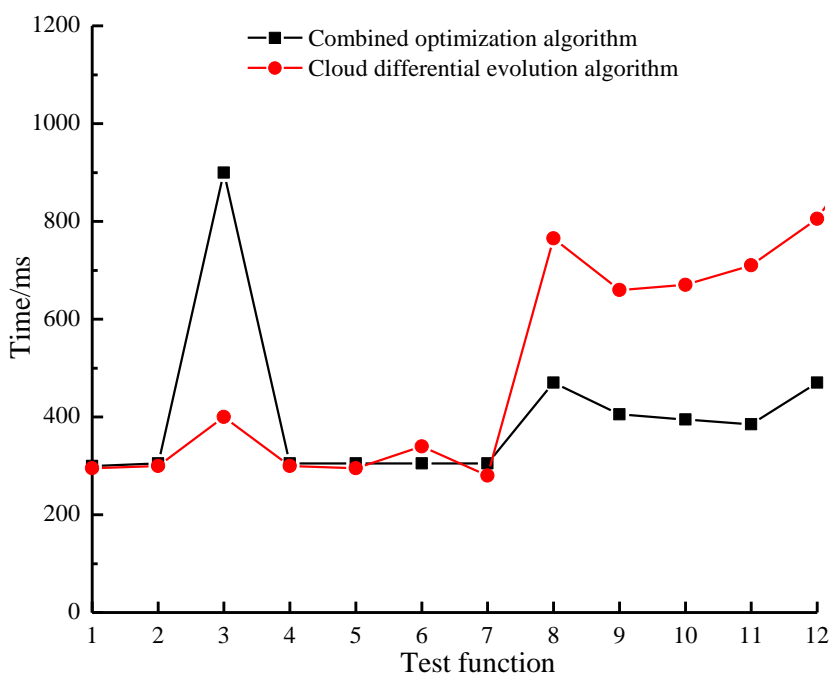

Figure 9. Comparison of average running time of test functions of two algorithms 


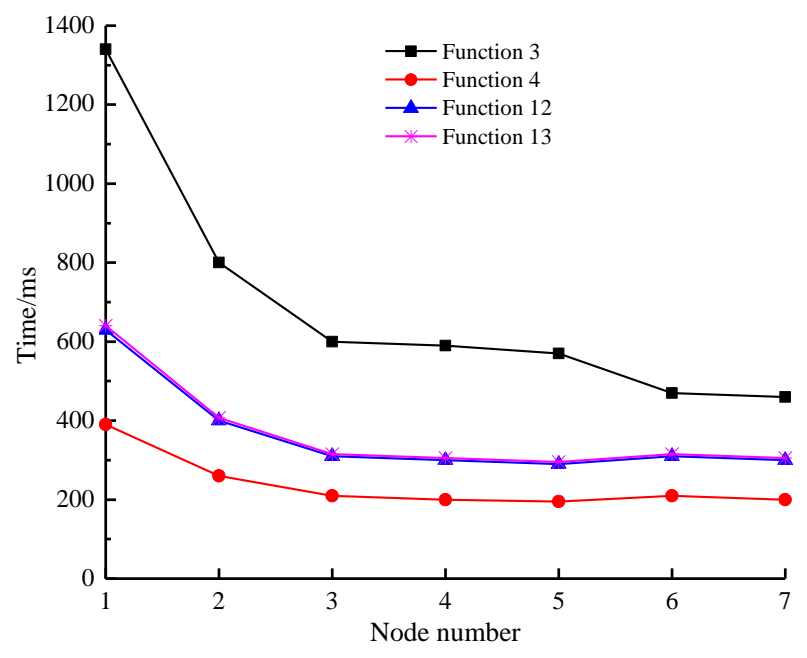

Figure 10. Comparison of test function running time under different nodes

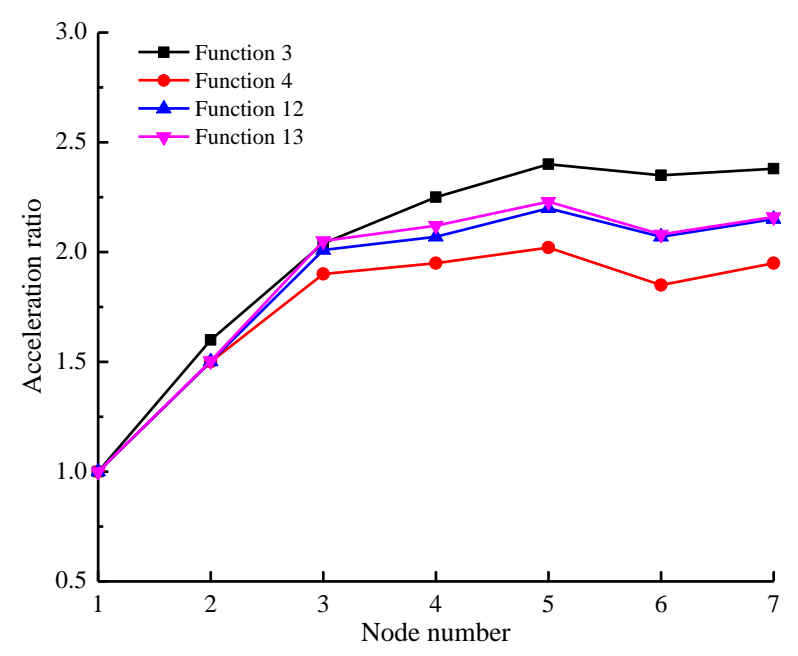

Figure 11. Acceleration ratio experiment result chart

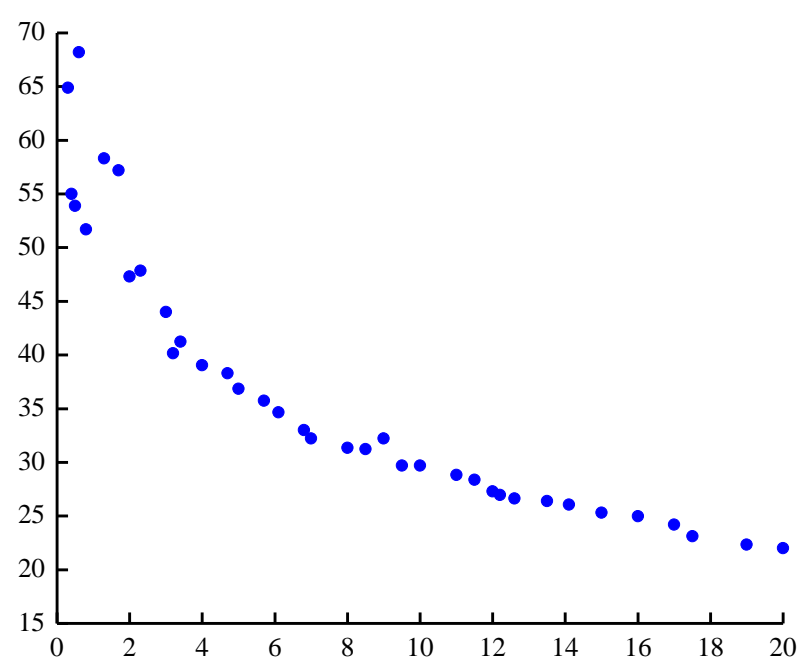

Figure 12. Improved service combination optimization algorithm simulation results

\section{CONCLUSIONS}

Based on COA algorithm, this paper studies the cloud intelligent logistics service and service combination method, and also the operation mechanism of CIL service. Then it proposes the solution method and algorithm of COA algorithm. The specific conclusions are as follows:

(1) The quality of service matching is directly related to the quality of CIL service. The use of combination optimization algorithm can find the extreme value of service matching and provide optimal solution.

(2) The time spent by the COA algorithm is significantly smaller than that of the clustering algorithm. The time spent on a single task is inversely proportional to the number of nodes. Increasing the number of nodes can improve the processing efficiency of the COA algorithm; as the data set increases, it takes longer with the same number of nodes.

(3) By comparing the COA algorithm and the cloud DE algorithm to solve the multi-objective optimization problem of CIL service combination. It's found that the COA algorithm is better than the cloud DE algorithm, and it has better stability and scalability.

\section{ACKNOWLEDGEMENT}

This paper is supported by National Natural Science Foundation of China (No.: 71371061): Research on Coordination of Customer-oriented Supply Chain Coopetition Network and Catastrophic Response; Humanities and Social Sciences Project of Ministry of Education of China (No.: 16YJA790024): Research on Compatible Incentive and Market Structure Evolution of Differentiated Platform.

\section{REFERENCES}

[1] Lin SM. (2013). Analysis of service satisfaction in web auction logistics service using; a combination of fruit fly optimization algorithm and general regression; neural network. Neural Computing \& Applications 22(3-4): 783-791. https://doi.org/10.1007/s00521-011-0769-1

[2] Teschemacher U, Reinhart G. (2017). Ant colony optimization algorithms to enable dynamic milk run logistics. Procedia CIRP 63: 762-767. https://doi.org/10.1016/j.procir.2017.03.125

[3] Wang Y, Ma X, Xu M, Liu Y, Wang Y. (2015). Twoechelon logistics distribution region partitioning problem based on a hybrid particle swarm optimization-genetic algorithm. Expert Systems with Applications an International Journal 42(12): 5019-5031. https://doi.org/10.1016/j.eswa.2015.02.058

[4] Walther L, Rizvanolli A, Wendebourg M, Jahn C. (2016) Modeling and optimization algorithms in ship weather routing. International Journal of e-Navigation and Maritime Economy 4: 31-45. https://doi.org/10.1016/j.enavi.2016.06.004

[5] Zhang Q, Jiang C, Zhang J, Wei Y. (2014). Application of genetic algorithm in functional area layout of railway logistics park. Procedia - Social and Behavioral Sciences 138: 269-278. https://doi.org/10.1016/j.sbspro.2014.07.204

[6] Wu M, Wang L, Li M, Long H. (2013). An approach based on the sir epidemic model and a genetic algorithm for optimizing product feature combinations in feature fatigue analysis. Journal of Intelligent Manufacturing 26(1): 1-11. https://doi.org/10.1007/s10845-013-0773-7

[7] Lee JE, Chung KY, Lee KD, Gen M. (2015). A multi- 
objective hybrid genetic algorithm to minimize the total cost and delivery tardiness in a reverse logistics. Multimedia Tools \& Applications 74(20): 9067-9085. https://doi.org/10.1007/s11042-013-1594-6

[8] Ibrahim RA, Elaziz MA, Lu S. (2018). Chaotic opposition-based grey-wolf optimization algorithm based on differential evolution and disruption operator for global optimization. Expert Systems with Applications 108: 1-27. https://doi.org/10.1016/j.eswa.2018.04.028

[9] Ndhaief N, Bistorin O, Rezg N. (2018). A modelling approach for city locating logistic platforms based on combined forward and reverse flows. IFAC-Papers OnLine 50(1): 11701-11706. https://doi.org/10.1016/j.ifacol.2017.08.1691

[10] Tang LX, Jiang W, Saharidis GKD. (2013). An improved benders decomposition algorithm for the logistics facility location problem with capacity expansions. Annals of Operations Research 210(1): 165-190. https://doi.org/10.1007/s10479-011-1050-9

[11] Talatahari S, Azar BF, Sheikholeslami R, Gandomi AH. (2012). Imperialist competitive algorithm combined with chaos for global optimization. Communications in Nonlinear Science \& Numerical Simulation 17(3): 13121319. https://doi.org/10.1016/j.cnsns.2011.08.021

[12] Chen CC, Schonfeld P. (2012). A hybrid heuristic technique for optimizing intermodal logistics timed transfer systems. Procedia - Social and Behavioral
Sciences

48(9):

2566-2576

https://doi.org/10.1016/j.sbspro.2012.06.1227

[13] Lin YK, Yeh CT. (2010). Optimal carrier selection based on network reliability criterion for stochastic logistics networks. International Journal of Production Economics 128(2): 510-517. https://doi.org/10.1016/j.ijpe.2010.07.001

[14] Qin J, Shi F, Miao LX, Tan GJ. (2009). Optimal model and algorithm for multi-commodity logistics network design considering stochastic demand and inventory control. Systems Engineering - Theory \& Practice 29(4): 176-183. https://doi.org/10.1016/S1874-8651(10)600191

[15] Yang M, Mahmood M, Zhou X, Shafaq S, Zahid L. (2017). Design and implementation of cloud platform for intelligent logistics in the trend of intellectualization. China Communications 14(10): 180-191. https://doi.org/10.1109/CC.2017.8107642

[16] Jiang FC, Hsu CH, Wang S. (2017). Logistic support architecture with petri net design in cloud environment for services and profit optimization. IEEE Transactions on Services Computing 10(6): 879-888. https://doi.org/10.1109/TSC.2016.2514506

[17] He W, Yan G, Xu LD. (2014). Developing vehicular data cloud services in the IoT environment. IEEE Transactions on Industrial Informatics 10(2): 1587-1595. https://doi.org/10.1002/bies.1082 